\title{
Protective Effects of S-Adenosylmethionine and Its Combinations With Taurine and/or Betaine Against Lipopolysaccharide or Polyinosinic-polycytidylic Acid-induced Acute Hepatotoxicity
}

\author{
Seo Yeon Lee, Kwang Suk Ko \\ Department of Nutritional Science and Food Management, Ewha Womans University, Seoul, Korea
}

\begin{abstract}
Background: Several mechanisms for the pathogenesis of many liver diseases are related with oxidative stress, endotoxins, and infections by many microorganisms. These can lead to chronic hepatitis, cirrhosis, and even liver cancer. The aim of this study was to evaluate the effects of S-adenosylmethionine (SAMe) and its combinations with taurine and/or betaine against hepatotoxicites induced by lipopolysaccharide (LPS) or polyinosinic-polycytidylic acid (polyl:C).

Methods: RAW 264.7 macrophage cells and seven-week-old male C57BL/6 mice were pretreated with SAMe (SAM or AdoMet), taurine, and/or betaine. In order to mimic hepatic injury like endotoxemia or viral infection, cells and mice were treated with LPS or polyl:C. Concentrations of glutathione (GSH), mRNA expressions of GSH synthesizing enzymes, and inflammatory markers were measured by biochemical assays and quantitative real-time PCR.

Results: In RAW 264.7 cells and mice, pretreatment of SAMe alone or SAMe with taurine and/or betaine attenuated the decrease in GSH levels and mRNA expressions of GSH synthesizing enzymes. In addition, pretreatment of SAMe with taurine and/or betaine prevented the excessive increase in inflammatory mediators produced by LPS or polyl:C treatment.

Conclusions: Treatment with SAMe in combination with taurine and betaine, would have anti-oxidant functions in addition to anti-inflammatory action against bacterial and/or viral inflammation.
\end{abstract}

(J Cancer Prev 2016;21:152-163)

Key Words: S-adenosylmethionine, Taurine, Betaine, Lipopolysaccharides, Polyinosinic-polycytidylic acid

\section{INTRODUCTION}

Eighty percent of the liver volume is hepatocytes that have a significant role in endotoxin detoxification. The remaining cells, such as liver endothelial cells and Kupffer cells, play an important role in innate immune defenses against pathogens and elimination of microorganisms. ${ }^{1}$ Infection with the hepatitis virus and innate immunity is associated with pathogenesis of liver cirrhosis and development of hepatocellular carcinoma (HCC). Hepatitis virus-induced inflammation is considered as major risk factor for $\mathrm{HCC}^{2}$

The formation of reactive metabolites triggers liver injury and activates an innate immune response, which in turn, causes inflammation. Since liver plays a major role in the removal of endotoxin, endotoxemia participates in the pathogenesis of liver diseases. ${ }^{3}$ Lipopolysaccharide (LPS) is an essential component of the outer layer of the membrane of gram-negative bacteria. ${ }^{4}$ Toll-like receptors (TLRs) recognize microbial molecules and initiate the inflammatory response. ${ }^{5}$ LPS is recognized by TLR4 which is expressed on macrophages and other cell types in the liver. ${ }^{4}$ LPS can trigger the production of inflammatory mediators, such as nitric oxide (NO) which is synthesized by inducible nitric oxide synthase (iNOS) in macrophages. ${ }^{3.6}$ In addition, several cytokines play important roles in mediating an inflammatory

Received August 8, 2016, Revised August 25, 2016, Accepted August 25, 2016

Correspondence to: Kwang Suk Ko

Department of Nutritional Science and Food Management, Ewha Womans University, 52 Ewhayeodae-gil, Seodaemun-gu, Seoul 03760, Korea

Tel: +82-2-3277-6859, Fax: +82-2-3277-2862, E-mail: kko@ewha.ac.kr, ORCID: Kwang Suk Ko, http://orcid.org/0000-0002-0515-5904

Copyright (C) 2016 Korean Society of Cancer Prevention

(c) This is an Open Access article distributed under the terms of the Creative Commons Attribution Non-Commercial License (http://creativecommons.org/licenses/by-nc/4.0) which permits unrestricted non-commercial use, distribution, and reproduction in any medium, provided the original work is properly cited. 
reaction, such as TNF- $\alpha$. These cytokines mediate some aspects of the response to infection and inflammation. ${ }^{7}$

In cells infected by RNA viruses, viral RNA replication produces double stranded RNA (dsRNA). ${ }^{8}$ TLR3 is found in cells such as macrophages and dendritic cells and recognizes dsRNA from viruses. ${ }^{9}$ When TLR3 recognizes pathogen-associated molecular patterns, it induces inflammatory responses. In hepatic immune disorders, TLR3 signaling pathways are activated. ${ }^{10}$ Polyinosinicpolycytidylic acid (polyI:C) is a synthetic analogue of dsRNA, which may induce the immune response similar to viral infection. ${ }^{11}$ Like other pathogenic endotoxins, dsRNA can trigger the generation of inflammatory cytokines and chemokines such as TNF- $\alpha{ }^{8}$ Cytokines are necessary for the immune system to communicate, but excessive and uncontrolled production causes serious systemic complications. ${ }^{12,13}$

An impairment of methionine metabolism is often shown in the liver diseases. ${ }^{14,15}$ This can lead to an improper metabolism of sulfur-containing amino acids (SCAA) and their metabolic substances including methionine, S-adenosylmethionine (SAMe, SAM or AdoMet), homocysteine, S-adenosylhomocysteine (SAH) and glutathione (GSH). ${ }^{16} \mathrm{GSH}$, a powerful antioxidant in liver, protects the liver from oxidative stress and regulates the inflammatory response. ${ }^{17}$ The progression of hepatitis may involve oxidative stress. ${ }^{18}$ The inactivation of methionine adenosyltransferase (MAT) decreases hepatic SAMe and GSH concentration in liver diseases. ${ }^{19}$ SAMe, which is the metabolite of a reaction catalyzed by MAT, is important for synthesizing $\mathrm{GSH}^{20}$

Taurine, an effective antioxidant, is synthesized from methionine and cysteine in liver. ${ }^{21}$ It has been shown that patients with liver disease have low serum cysteine, taurine and GSH concentration..$^{22,23}$ Nearly half of the cysteine is converted to GSH, and rest of that is converted into taurine and participates in protein synthesis depending on the need of the cells. ${ }^{24}$

Betaine is a metabolite of choline. ${ }^{25}$ Betaine is involved in remethylation by donating its methyl group to homocysteine, which is then converted to methionine by betaine-homocysteine methyltransferase. ${ }^{25}$ Therefore, betaine can restore the hepatic methionine level and regulate the SAM:SAH ratio. ${ }^{26}$ If the SAM:SAH ratio is decreased, it could decrease methylation by lowering the activation of methyltransferases. ${ }^{26}$ Betaine supplementation may enhance trans-sulfuration by increasing methionine and SAMe in liver. ${ }^{27}$ Moreover, betaine may have an antioxidative effect by maintaining SAMe levels and preventing the elevation of homocysteine. ${ }^{25}$

GSH is an important factor determining the susceptibility of the liver to damage induced by LPS. ${ }^{28}$ SAMe has been shown to restore the GSH concentration. ${ }^{20}$ Taurine and betaine are also vital compounds in SCAA metabolism. The aim of this study is to evaluate the effects of SAMe, taurine, betaine and their combinations on GSH homeostasis and inflammatory mediators in LPS- or polyI:C-treated RAW 264.7 cells and C57BL/6 mice.

\section{MATERIALS AND METHODS}

\section{Materials}

SAMe in the form of S-adenosyl-L-methionine disulfate $\mathrm{p}$ toluensulfonate (SAMe PATES) dried powder was obtained from Samoh Pharm (Seoul, Korea). Taurine, betaine, LPS (Escherichia coli 055:B5), and polyI:C were purchased from Sigma Chemical (St. Louis, MO, USA).

\section{Cell culture}

RAW 264.7, a murine macrophage cell line, was obtained from the Korean Cell Line Bank (Seoul, Korea). RAW 264.7 cells were maintained in Dulbecco's modified Eagle's medium (Wel Gene, Daegu, Korea) supplemented with 10\% FBS (v/v) (Hyclone, Logan, UT, USA), $100 \mathrm{U} / \mathrm{mL}$ penicillin and $100 \mu \mathrm{g} / \mathrm{mL}$ streptomycin (Hyclone) at $37^{\circ} \mathrm{C}$ in a $5 \% \mathrm{CO}_{2}$ humidified incubator.

\section{Cell treatment}

RAW 264.7 cells (passage numbers: 10-18) were seeded on 6-well plates $\left(8.5 \times 10^{5}\right.$ cells/well) and incubated. After 6 hours, cells were pretreated with SAMe $(0.5 \mathrm{mM})$, taurine $(10 \mathrm{mM})$ and/or betaine $(1 \mathrm{mM})$ and incubated for 16 hours. LPS and polyI: $\mathrm{C}$ were suspended in PBS. PolyI: $\mathrm{C}$ was heated for 10 minutes at $65^{\circ} \mathrm{C}$ and cooled for 1 hour at room temperature to achieve re-annealing of the reconstitution. After pretreatment, the cells were stimulated with LPS $(0.5 \mu \mathrm{g} / \mathrm{mL})$ or polyI:C $(10 \mu \mathrm{g} / \mathrm{mL})$ for 4 hours. These concentrations of LPS and polyI: $\mathrm{C}$ were shown to increase the expression of pro-inflammatory mediators in RAW 264.7 cells in other experiments. ${ }^{29.30}$

\section{Cell viability}

RAW 264.7 cells were seeded in 96-well plates $\left(0.25 \times 10^{5}\right.$ cells/well) and incubated at $37^{\circ} \mathrm{C}$ in a $5 \% \mathrm{CO}_{2}$ environment. After 6 hours, cells were pretreated with SAMe $(0.5 \mathrm{mM})$, taurine (10 $\mathrm{mM})$ and/or betaine $(1 \mathrm{mM})$ and incubated for 16 hours. After pretreatment, they were stimulated with LPS $(0.5 \mu \mathrm{g} / \mathrm{mL})$ or polyI:C (10 $\mu \mathrm{g} / \mathrm{mL})$ for 4 hours. Then, each well was inoculated with MTT reagent (Sigma-Aldrich, St. Louis, MO, USA) and incubated at $37^{\circ} \mathrm{C}$ for 2 hours. The supernatant was gently 
removed, and $100 \mu \mathrm{L}$ of dimethyl sulfoxide was added into each well. The absorbance of each well was measured at $560 \mathrm{~nm}$ using a microplate reader (Molecular Devices, Sunnyvale, CA, USA).

\section{Animal experiments}

Five-week-old male C57BL/6 mice were purchased from Samtako (Osan, Korea). They were maintained at $25^{\circ} \mathrm{C} \pm 3^{\circ} \mathrm{C}$ with a 12:12-hour light-dark cycle, and given chow (Altromin, Lage, Germany) and deionized water. The mouse chow contains 12 $\mathrm{mg} / \mathrm{kg}$ of vitamin B2, $24 \mathrm{mg} / \mathrm{kg}$ of vitamin B6, $24 \mu \mathrm{g} / \mathrm{kg}$ of vitamin B12, $2 \mathrm{mg} / \mathrm{kg}$ of folate, $600 \mathrm{mg} / \mathrm{kg}$ of choline chloride and $0.7 \%$ of methionine and cysteine. After acclimation for 10 days, the mice were randomly divided into fifteen groups ( $n=5$-6/group) as follows: control, only LPS or polyI:C and LPS or polyl:C plus SAMe, taurine, betaine, SAMe with taurine, SAMe with betaine or SAMe with taurine and betaine. Control, LPS and polyl:C groups were administered $0.1 \mathrm{~mL} / \mathrm{kg}$ body weight (BW) PBS. SAMe, taurine and betaine were freshly dissolved in PBS. SAMe-treated mice were given $100 \mathrm{mg} / \mathrm{kg}$ BW. Taurine-treated mice were given $200 \mathrm{mg} / \mathrm{kg}$ BW. Betaine-treated mice were given $500 \mathrm{mg} / \mathrm{kg}$ BW every day for a week by intragastric gavage. Six hours after the last pretreatment, LPS was injected intraperitoneally (i.p.) $15 \mathrm{mg} / \mathrm{kg}$ BW to LPS groups. Polyl:C was injected $50 \mathrm{mg} / \mathrm{kg}$ BW (i.p.) to polyI:C groups. After exposure to LPS or polyl:C for 18 hours, animals were sacrificed.

The experimental procedures were approved by the Institutional Animal Care and Use Committee (IACUC) at Ewha Womans University (approval number 15-059).

\section{Serum alanine aminotransferase and aspartate aminotransferase}

Alanine aminotransferase (ALT) and aspartate aminotransferase (AST) activities were examined using kits (Asan Pharm, Hwaseong, Korea) based on Retiman-Frankel method. ${ }^{31}$

\section{Glutathione concentration}

GSH concentration was measured by using GSH reductase (Sigma-Aldrich). Liver was homogenized in PBS and cell was scrapped with PBS. Homogenates were centrifuged at 10,000 $\times g$ for 30 minutes at $4^{\circ} \mathrm{C}$. A $0.1 \mathrm{~mL}$ aliquot of supernatant was added to the same volume of $0.6 \mathrm{M}$ perchloric acid (Junsei Chemical, Tokyo, Japan), and the GSH concentration determined. The 0.1 $\mathrm{mL}$ GSH standards and samples (Sigma-Aldrich) were added to 2.5 $\mathrm{mL}$ reaction buffer (0.15 mM NADPH [Sigma-Aldrich), $0.1 \mathrm{mM}$ 5.5'-dithio-bis-(2-nitrobenzoic acid) [Sigma-Aldrich], $50 \mathrm{mM}$ $\mathrm{NaPO}_{4}$ [Junsei Chemical], $1.5 \mathrm{mM}$ ethylenediaminetetraacetic acid [E5124; Sigma-Aldrich] and $0.1 \mathrm{~mL}$ GSH reductase [10 units/mL]). Changes in absorbance for a 1 minute period were measured at $412 \mathrm{~nm}$ using a Biochrom Libra S50 spectrophotometer (Biochrom Ltd., Cambridge, UK). The concentrations of GSH were calculated as $\mathrm{nmol} / \mathrm{mg}$ protein.

\section{RNA isolation and quantitative real-time reverse transcriptase-PCR}

Total RNA was extracted from liver tissues and cells using Trizol (Life Technologies, Grand Island, NY, USA) according to the manufacturer's instructions. RNA was reverse transcribed into complementary DNA (CDNA) using a first strand CDNA synthesis kit (Thermo Fisher Scientific, Waltham, MA, USA). Quantitative real time PCR (qPCR) was performed using Maxima SYBR Green qPCR Master Mixes (Thermo Fisher Scientific). qPCR was conducted in duplicate with the Rotor Gene $\mathrm{Q}$ machine (Qiagen, Hilde, German). Amplification was performed by starting with a template denaturation step at $95^{\circ} \mathrm{C}$ for 10 minutes, followed by 40 cycles at $95^{\circ} \mathrm{C}$ for 15 seconds and $60^{\circ} \mathrm{C}$ for 1 minute. The primer sequences used for this experiment are as follows: glutamate-cysteine ligase catalytic subunit (NM_010295, GCLC): 5'-ACA CCT GGA TGA TGC CAA CGA G-3' (forward), 5'-CCT CCA TTG GTC GGA ACT CTA C-3' (reverse), glutamate-cysteine ligase modifier subunit (NM_008129, GCLM): 5'-TCC TGC TGT GTG ATG CCA CCA G-3' (forward), 5'-GCT TCC TGG AAA CTT GCC TCA G-3' (reverse), GSH synthase (NM_0008180, GS): 5'-CCA GGA AGT TGC TGT GGT GTA C-3' (forward), 5'-GCT GTA TGG CAA TGT CTG GAC AC-3' (reverse), hypoxanthine guanine phosphoribosyl transferase (NM_013556, Hprt1): 5'-CAG GCC AGA CTT TGT TGG AT-3' (forward), 5'-TTG CGC TCA TCT TAG GCT TT-3' (reverse), TNF- $\alpha$ (NM_001278601): 5'-GGT GCC TAT GTC TCA GCC TCT T-3' (forward), 5'-GCC ATA GAA CTG ATG AGA GGG AG-3' (reverse), iNOS (NM_010927, iNOS): 5'-GAG ACA GGG AAG TCT GAA GCA C-3' (forward), 5'-CCA GCA GTA GTT GCT CCT CTT C-3' (reverse). The relative amounts of these mRNAs were normalized to the amounts of hprtl and the relative amounts of RNAs were calculated using the comparative CT method. All data are expressed as a relative quantity to each control value.

\section{Nitric oxide assay}

The production of NO, which is a major macrophage-derived inflammatory mediator, was evaluated by measuring the accumulated nitrite content in culture medium and serum. RAW 264.7 cells (passage numbers: 10-18) were seeded on 6-well plates $\left(8.5 \times 10^{5} \mathrm{cells} /\right.$ well $)$ and incubated for 6 hours. Cells were then treated with SAMe $(0.5 \mathrm{mM})$, taurine $(10 \mathrm{mM})$ and/or betaine $(1$ 
$\mathrm{mM}$ ) and incubated for 16 hours. After this incubation, they were stimulated with LPS $(0.5 \mu \mathrm{g} / \mathrm{mL})$ or polyI:C $(10 \mu \mathrm{g} / \mathrm{mL})$ and incubated overnight. The cell culture media were collected and centrifuged at $1,500 \mathrm{rpm}$ for 10 minutes at $4^{\circ} \mathrm{C}$. Griess reagent $(1 \%$ sulfanilamide in $5 \%$ phosphoric acid and $0.1 \% \mathrm{~N}$-(1-Naphthyl)ethylenediamine dihydrochloride in water) was added to each well in a 1:1 (v/v) manner. After gentle mixing, the plates were incubated in a dark room for 15 minutes and the absorbance was measured at $540 \mathrm{~nm}$ using a microplate reader (Molecular Devices). The nitrite concentration was calculated using a standard curve by serial dilutions of standard nitrite solutions (sodium nitrite in deionized water).

\section{Measurement of TNF- $\alpha$ in the serum}

TNF- $\alpha$ level in serum was determined using an ELISA kits ( $R \& D$ Systems, Minneapolis, MN, USA).

\section{Statistical analysis}

Results are expressed as the means \pm SEM. The data were analyzed using the Statistical Analysis Systems package ver. 9.3 (SAS Institute, Cary, NY, USA). The differences among groups were analyzed by one-way ANOVA with post-hoc Duncan's test. $P$-values less than 0.01 were considered statistically significant.

\section{RESULTS}

1. Effects of S-adenosylmethionine, taurine, betaine, lipopolysaccharide, and polyinosinic-polycytidylic acid on the viability of RAW 264.7 cells

To examine the effect of SAMe, taurine, betaine, LPS and polyI:C on cell viability, MTT assay was performed (data are not shown). The viability was more than $90 \%$ in all groups. The results indicate that $0.5 \mathrm{mM}$ of SAMe, $10 \mathrm{mM}$ of taurine, $1 \mathrm{mM}$ of betaine, $0.5 \mu \mathrm{g} / \mathrm{mL}$ of LPS and $10 \mu \mathrm{g} / \mathrm{mL}$ of polyl:C are not cytotoxic to RAW 264.7 cells.

2. Effects of S-adenosylmethionine,taurine, and/or betaine on intracellular GSH levels, mRNA levels of GSH synthesizing enzymes and inflammation markers in lipopolysaccharide-treated RAW 264.7 cells

LPS treatment had a tendency to lower the GSH level (Fig. 1A). Pretreatment of SAMe and its combinations with taurine and/or betaine attenuated the decrease in GSH level during endotoxemia. GSH level was increased by nearly 1.5 fold in SAMe alone-treated cells than in control cells. SAMe pretreatment significantly attenuated the decrease in mRNA levels of GSH synthesizing enzymes in RAW 264.7 cells (Fig. 1B). In contrast, only taurine or betaine treatment had no significant effect on mRNA levels of GSH synthesizing enzymes. SAMe and taurine combination, with or without betaine, also attenuated significantly the decrease in GCLC and GCLM mRNA levels.

Pretreatment of cells with SAMe, taurine and/or betaine significantly inhibited excessive production of NO in RAW 264.7 cells stimulated by LPS (Fig. 1C). Since NO production is related to the expression of iNOS, mRNA expression level was measured (Fig. 1D). iNOS mRNA level was markedly upregulated by LPS treatment but pretreatment with either taurine or betaine dramatically inhibited upregulation of iNOS expression induced by LPS. To measure production of proinflammatory cytokine, TNF- $\alpha$ mRNA expression levels were measured. Stimulation of RAW 264.7 cells with LPS $(0.5 \mu \mathrm{g} / \mathrm{mL})$ for 4 hours markedly increased the TNF- $\alpha$ mRNA level (Fig. 1D). Pretreatment with SAMe, taurine, betaine and their combinations inhibited the increase in the LPS-induced TNF- $\alpha$ mRNA levels.

3. Effects of S-adenosylmethionine, taurine, and/or betaine on intracellular GSH levels, mRNA levels of GSH synthesizing enzymes and inflammation markers in polyinosinic-polycytidylic acid-treated RAW 264.7 cells

Polyl:C treatment also indicated a tendency to lower the GSH level (Fig. 2A). Interestingly, pretreatment with SAMe and its combinations with taurine prevented the decrease in GSH level in polyI:C-treated RAW 264.7 cells. Pretreatment with SAMe and its combinations with taurine and/or betaine significantly attenuated the decrease in mRNA levels of GSH synthesizing enzymes in polyI:C-treated RAW 264.7 cells (Fig. 2B). On the other hand, only taurine or betaine treatment had no significant effect on mRNA levels of GSH synthesizing enzymes.

RAW 264.7 cells treated with polyI:C alone slightly increased NO production compared to cells pretreated with SAMe and its combinations (Fig. 2C). Also, RAW 264.7 cells treated with polyl:C $(10 \mu \mathrm{g} / \mathrm{mL})$ for 4 hours caused higher levels of iNOS and TNF- $\alpha$ mRNA. Pretreatment with either taurine or betaine significantly inhibited the increase in the polyl:C-induced iNOS and TNF- $\alpha$ mRNA expressions (Fig. 2D). 

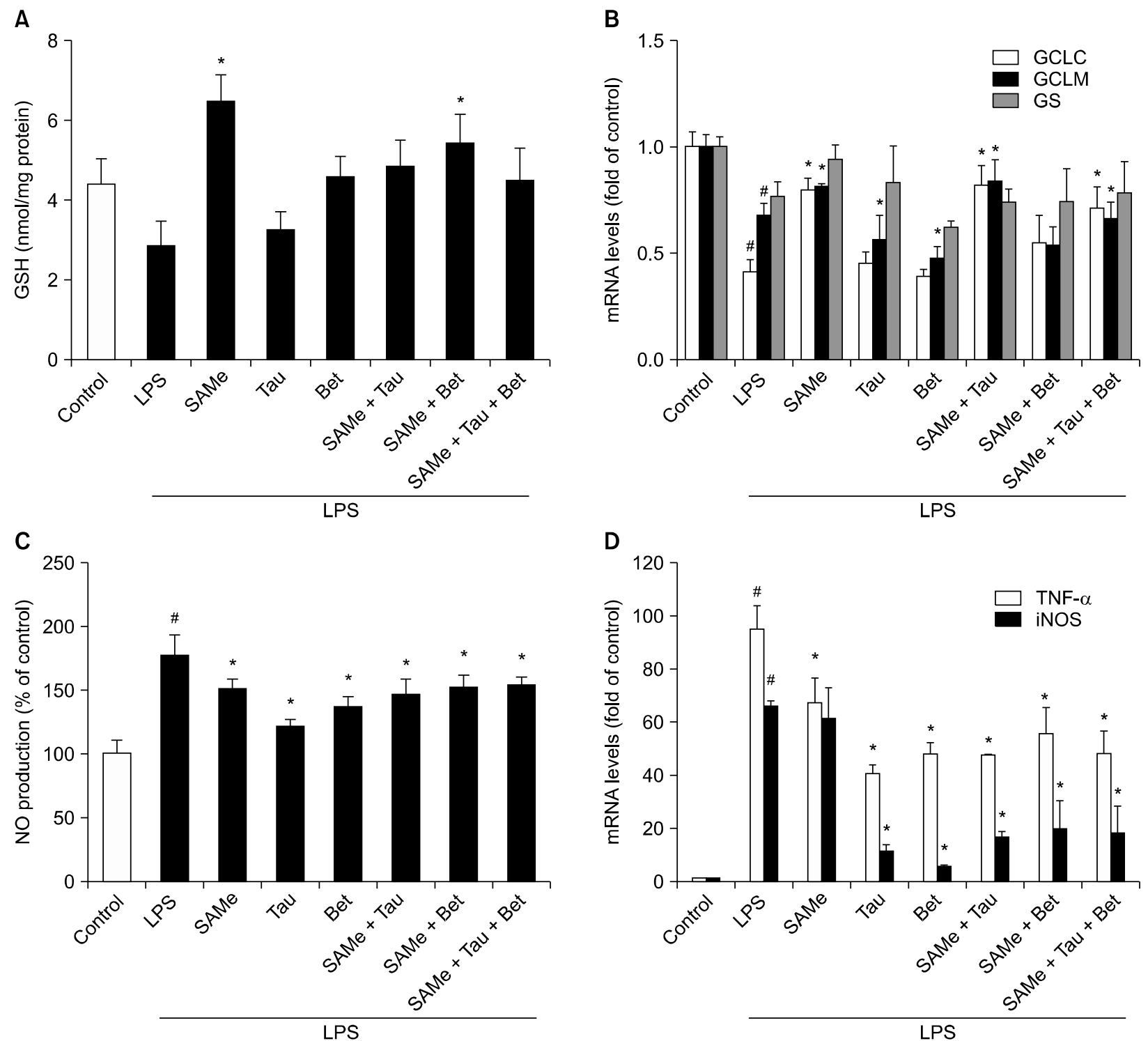

Figure 1. Effects of S-adenosylmethionine, taurine, betaine and their combinations on intracellular GSH levels and mRNA levels of GSH synthesizing enzymes and inflammation markers in lipopolysaccharide-treated RAW 264.7 cells. (A) Intracellular GSH levels were measured using glutathione reducase in LPS-stimulated RAW 264.7 cells. (B) mRNA levels of GCLC, GCLM and GS were measured using RT-qPCR. (C) NO production was measured from cell culture medium by the Griess reaction assay. (D) TNF- $\alpha$ and iNOS mRNA levels were measured by RT-qPCR and Hprt1 was used as the housekeeping gene. Cells were pretreated with SAMe (0.5 mM), Tau (10 mM), and/or Bet (1 mM) for 18 hours. After pretreatment, the cells were stimulated with LPS $(0.5 \mu \mathrm{g} / \mathrm{mL})$ for 4 hours. Values represent mean with SEM of three independent experiments. SAMe, S-adenosylmethionine; Tau, taurine; Bet, betaine; GSH, glutathione; LPS, lipopolysaccharide; GCLC, glutamate-cysteine ligas $\epsilon$ catalytic subunit; GCLM, glutamate-cysteine ligase modifier subunit; GS, GSH synthase; RT-qPCR, quantitative real-time reverse transcriptase-PCR; NO, nitric oxide; iNOS, inducible nitric oxide synthase. ${ }^{\#} P<0.01$ vs. control cells, ${ }^{*} P<0.01$ vs. LPS-treated group.

4. Effects of S-adenosylmethionine, taurine, betaine, lipopolysaccharide and polyinosinic-polycytidylic acid on body weight and liver weight mice

The BWs and liver weights were measured to determine the effects of administration of SAMe, taurine, betaine on injection of LPS and polyI:C (Table 1). LPS or polyI:C-treated groups tended to have higher ratios of liver weight (LW) to BW compared to the control group. The increase in LW may be associated with an increase in liver metabolic activity imperative for detoxification. 

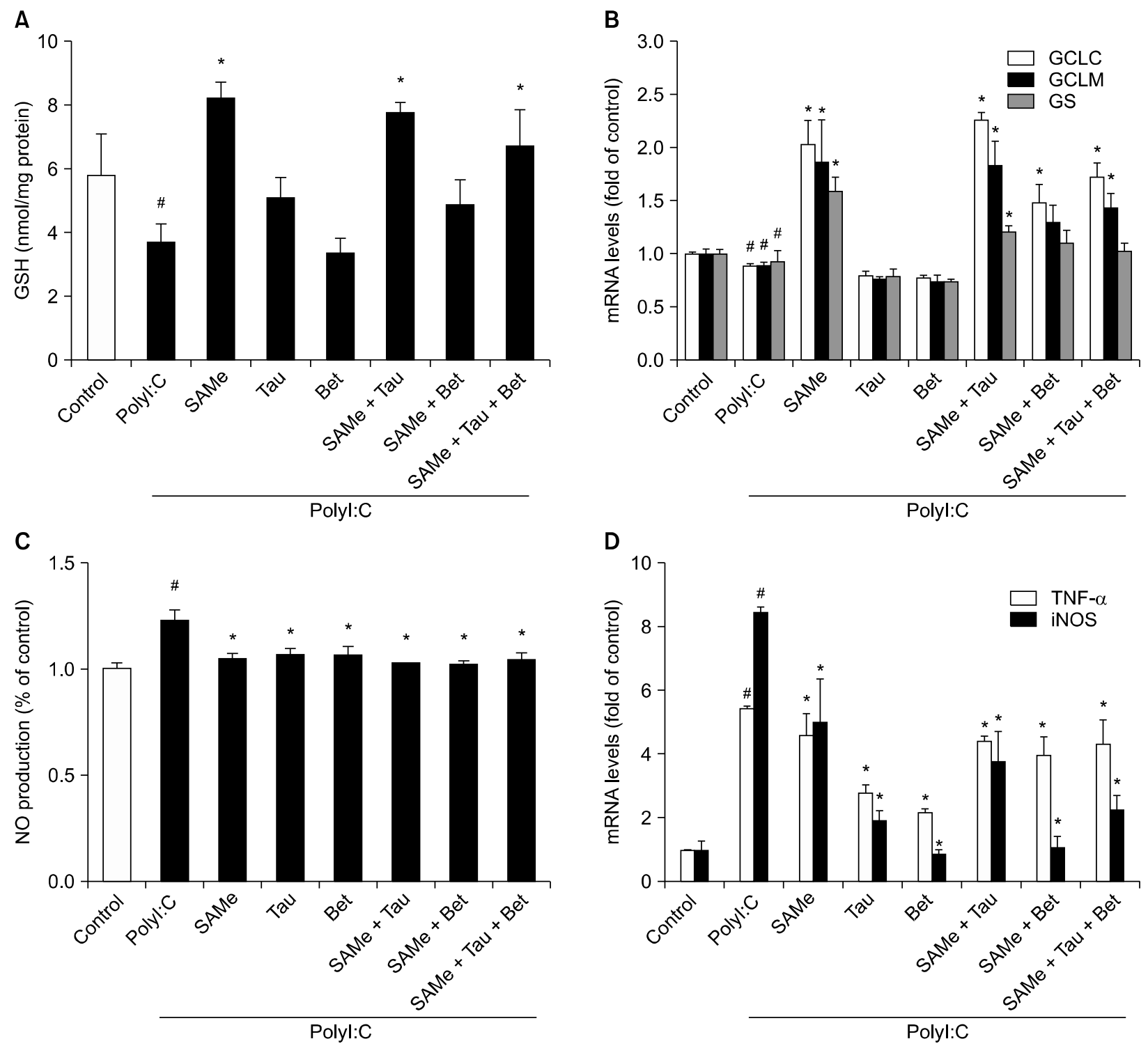

Figure 2. Effects of S-adenosylmethionine, taurine, betaine, and their combinations on intracellular GSH levels and mRNA levels of GSH synthesizing enzymes and inflammation markers in polyI:C-treated RAW 264.7 cells. (A) Intracellular GSH levels were measured using glutathione reductase in polyl:C-activated RAW 264.7 cells. (B) GCLC, GCLM, and GS mRNA levels were measured using RT-qPCR. (C) NO production was measured from cell culture medium by the Griess reaction assay. (D) TNF- $\alpha$ and iNOS mRNA levels were measured by RT-qPCR and Hprt1 was used as the housekeeping gene. Cells were pretreated with SAMe $(0.5 \mathrm{mM})$, Tau $(10 \mathrm{mM})$, and/or Bet (1 mM) for 18 hours. After pretreatment, the cells were stimulated with polyI:C $(10 \mu \mathrm{g} / \mathrm{mL})$ for 4 hours. Values represent mean with SEM of three independent experiments. SAMe, S-adenosylmethionine; Tau, taurine; Bet, betaine; GSH, glutathione; Polyl:C, polyinosinic-polycytidylic acid; GCLC, glutamate-cysteine ligase catalytic subunit; GCLM, glutamate-cysteine ligase modifier subunit; GS, GSH synthase; RT-qPCR, quantitative real-time reverse transcriptase-PCR; NO, nitric oxide; iNOS, inducible nitric oxide synthase. ${ }^{\#} P<0.01$ vs. control cells, $* P<0.01$ vs. PolyI:C-treated cells.

\section{Serum alanine aminotransferase and aspartate aminotransferase levels in mice}

Serum ALT and AST levels were measured to determine the level of liver injury (Fig. 3A, 4A). LPS- and polyl:C-treated groups showed significantly higher serum ALT and AST levels compared with their control groups. SAMe, taurine and betaine pretreatment attenuated the increase in serum ALT and AST levels induced by LPS or polyI:C treatment compared with only LPS- or polyI:C-treated group. 
Table 1. Effects of SAMe, taurine, betaine, and the combinations of three sulfur containing amino acids on body weight and liver weight in experimental mice

\begin{tabular}{|c|c|c|c|c|}
\hline Group & Treatments & BW (g) & LW (g) & $\mathrm{LW} / \mathrm{BW}$ ratio \\
\hline Control & Saline & $22.28 \pm 0.68$ & $0.85 \pm 0.05^{\mathrm{a}}$ & $0.38 \pm 0.02^{\mathrm{a}}$ \\
\hline \multirow[t]{7}{*}{ LPS } & Saline & $22.10 \pm 0.45$ & $1.13 \pm 0.03^{\mathrm{cd}}$ & $0.51 \pm 0.01^{\mathrm{d}}$ \\
\hline & SAMe & $22.52 \pm 1.27$ & $1.15 \pm 0.07^{\mathrm{cd}}$ & $0.51 \pm 0.02^{\mathrm{d}}$ \\
\hline & Tau & $22.70 \pm 0.97$ & $1.16 \pm 0.05^{\mathrm{d}}$ & $0.51 \pm 0.02^{\mathrm{d}}$ \\
\hline & Bet & $21.00 \pm 0.65$ & $0.96 \pm 0.05^{\mathrm{ab}}$ & $0.46 \pm 0.02^{\mathrm{b}}$ \\
\hline & $\mathrm{SAMe}+\mathrm{Tau}$ & $21.07 \pm 1.08$ & $1.06 \pm 0.08^{\mathrm{cd}}$ & $0.50 \pm 0.03^{\mathrm{cd}}$ \\
\hline & SAMe + Bet & $21.29 \pm 0.30$ & $1.03 \pm 0.05^{\mathrm{bc}}$ & $0.48 \pm 0.02^{\mathrm{bcd}}$ \\
\hline & $\mathrm{SAMe}+\mathrm{Tau}+$ Bet & $21.03 \pm 1.19$ & $0.99 \pm 0.07^{\mathrm{b}}$ & $0.47 \pm 0.01^{b c}$ \\
\hline \multirow[t]{7}{*}{ PolyI:C } & Saline & $22.25 \pm 0.34$ & $0.97 \pm 0.02^{\mathrm{b}}$ & $0.44 \pm 0.01$ \\
\hline & SAMe & $22.59 \pm 0.61$ & $0.97 \pm 0.04^{\mathrm{b}}$ & $0.43 \pm 0.02$ \\
\hline & Tau & $22.19 \pm 0.78$ & $0.97 \pm 0.06^{\mathrm{a}}$ & $0.44 \pm 0.01$ \\
\hline & Bet & $22.71 \pm 0.70$ & $0.99 \pm 0.04^{\mathrm{b}}$ & $0.44 \pm 0.01$ \\
\hline & $\mathrm{SAMe}+\mathrm{Tau}$ & $22.43 \pm 1.12$ & $0.98 \pm 0.05^{\mathrm{b}}$ & $0.44 \pm 0.01$ \\
\hline & $\mathrm{SAMe}+\mathrm{Bet}$ & $22.57 \pm 0.43$ & $0.93 \pm 0.09^{\mathrm{a}}$ & $0.41 \pm 0.04$ \\
\hline & SAMe + Tau + Bet & $21.91 \pm 0.61$ & $0.94 \pm 0.05^{\mathrm{a}}$ & $0.43 \pm 0.01$ \\
\hline
\end{tabular}

Values are presented as mean \pm SEM $(n=5)$. SAMe, S-adenosylmethionine; Tau, taurine; Bet, betaine; BW, body weight; LW, liver weight: LPS, lipopolysaccharide; PolyI:C, polyinosinic-polycytidylic acid. abcd Values with different superscripted alphabets are significantly different at $P<0.01$ level compared with control group.

6. Effects of S-adenosylmethionine and its combinations on hepatic glutathione levels, mRNA levels of glutathione synthesizing enzymes and inflammatory mediators in lipopolysaccharideinjected mice

Hepatic GSH concentrations were decreased in only LPS-treated mice compared to control mice. SAMe, taurine and/or betaine pretreatment significantly inhibited the decreases in hepatic GSH levels of LPS-treated mice (Fig. 3B). The mRNA levels of the GCLC, GCLM, and GS were decreased 18 hours after LPS recovered pretreatment of SAMe and its combinations with taurine and/or betaine (Fig. 3C). Pretreatment with SAMe combinations significantly inhibited LPS-induced production of NO and TNF- $\alpha$ (Fig. 3D) and LPS-induced mRNA expression of TNF- $\alpha$ and iNOS (Fig. 3E).

7. Effects of S-adenosylmethionine and its combinations on hepatic glutathione levels, mRNA levels of glutathione synthesizing enzymes and inflammatory mediators in polyl:C-injected mice

Hepatic GSH levels were slightly but not significantly decreased in only polyI:C-treated mice compared to control mice. However, SAMe and its combinations with taurine and/or betaine preserved GSH levels equal to those of control mice (Fig. 4B). The mRNA levels of the GCLC, GCLM, and GS were reduced 18 hours after polyI:C injection (Fig. 4C). SAMe and its combinations with taurine and/or betaine blocked the decreases in mRNA level of
GCLC, GCLM, and GS.

Pretreatment with SAMe combinations inhibited excessive production of TNF- $\alpha$ in serum induced by polyI:C (Fig. 4D). Serum NO was slightly increased in the group treated with only polyI:C but pretreatment with SAMe and its combinations did not result in increase in serum NO (Fig. 4D). Pretreatment of SAMe combinations with taurine and/or betaine more inhibited upregulation of TNF- $\alpha$ and iNOS mRNA than pretreatment with SAMe alone in polyl:C-injected mice (Fig. 4E).

\section{DISCUSSION}

The liver has an important role in detoxification and in the immune response. Liver damage induced by LPS or viral infection can lead to cirrhosis, hepatitis and liver cancer. ${ }^{15}$ Viral infection and innate immunity is associated with pathogenesis of liver cirrhosis and development of HCC. Virus-induced inflammation could be main risk factor for HCC. ${ }^{2}$ Impairment in methionine metabolism has been found in patients with liver disease. ${ }^{14,18}$ Alterations in the metabolism of sulfur-containing amino acids in liver could be related to depletion of SAMe and GSH concentration. ${ }^{32}$ In this work, we focused on the SAMe level in the liver to regulate the methionine metabolism and to ameliorate the depletion of GSH concentration. Some studies suggested that SAMe and betaine may ameliorate liver damage by maintaining normal methionine metabolism. ${ }^{18}$ It has also been shown that taurine administration could decrease oxidative stress. ${ }^{33}$ Given the importance of these compounds in methionine metabolism 
A

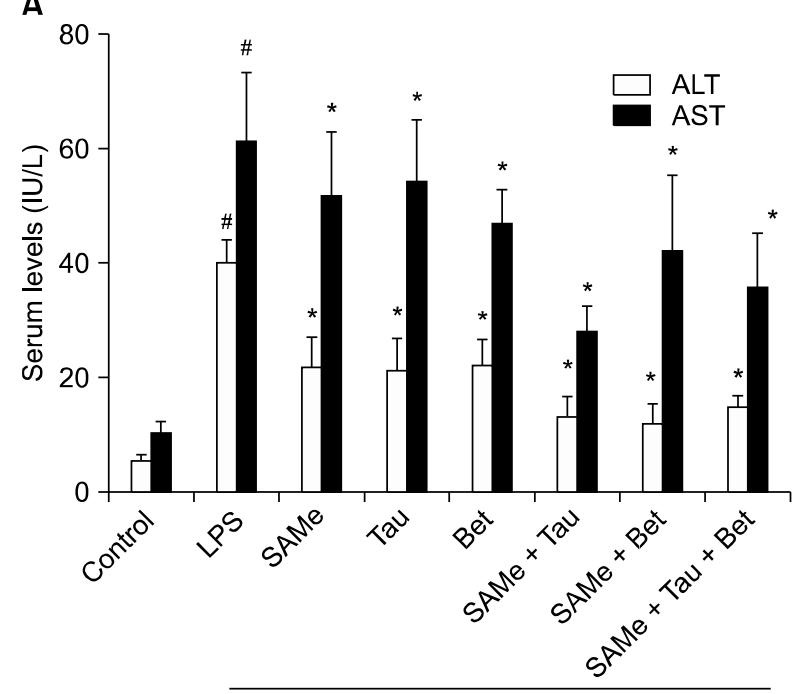

C

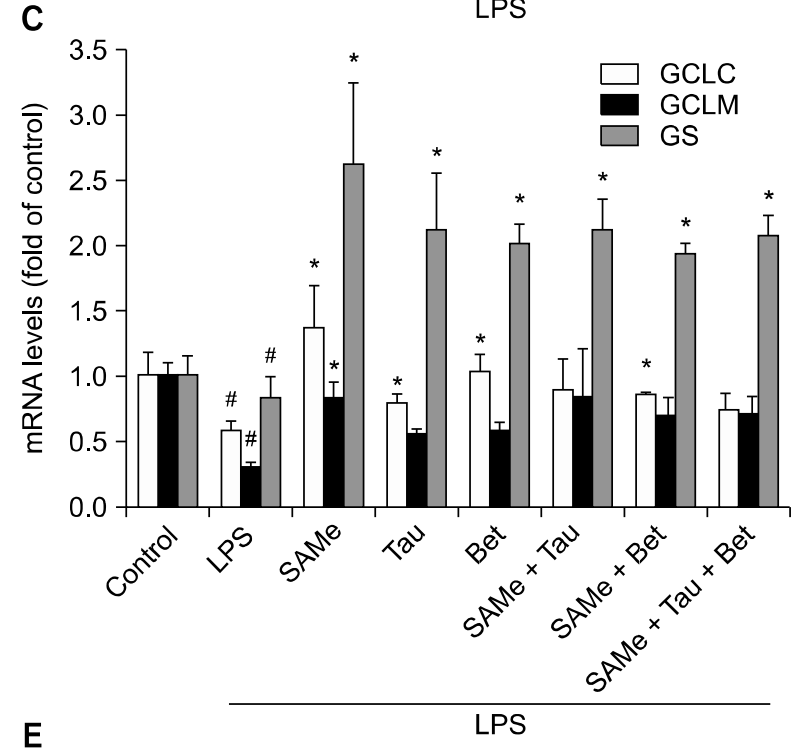

E

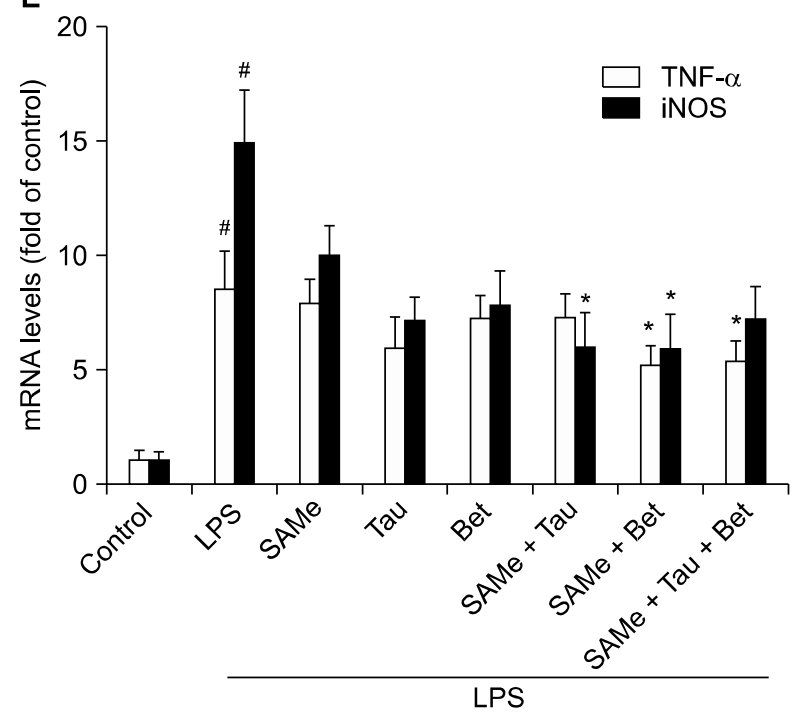

B
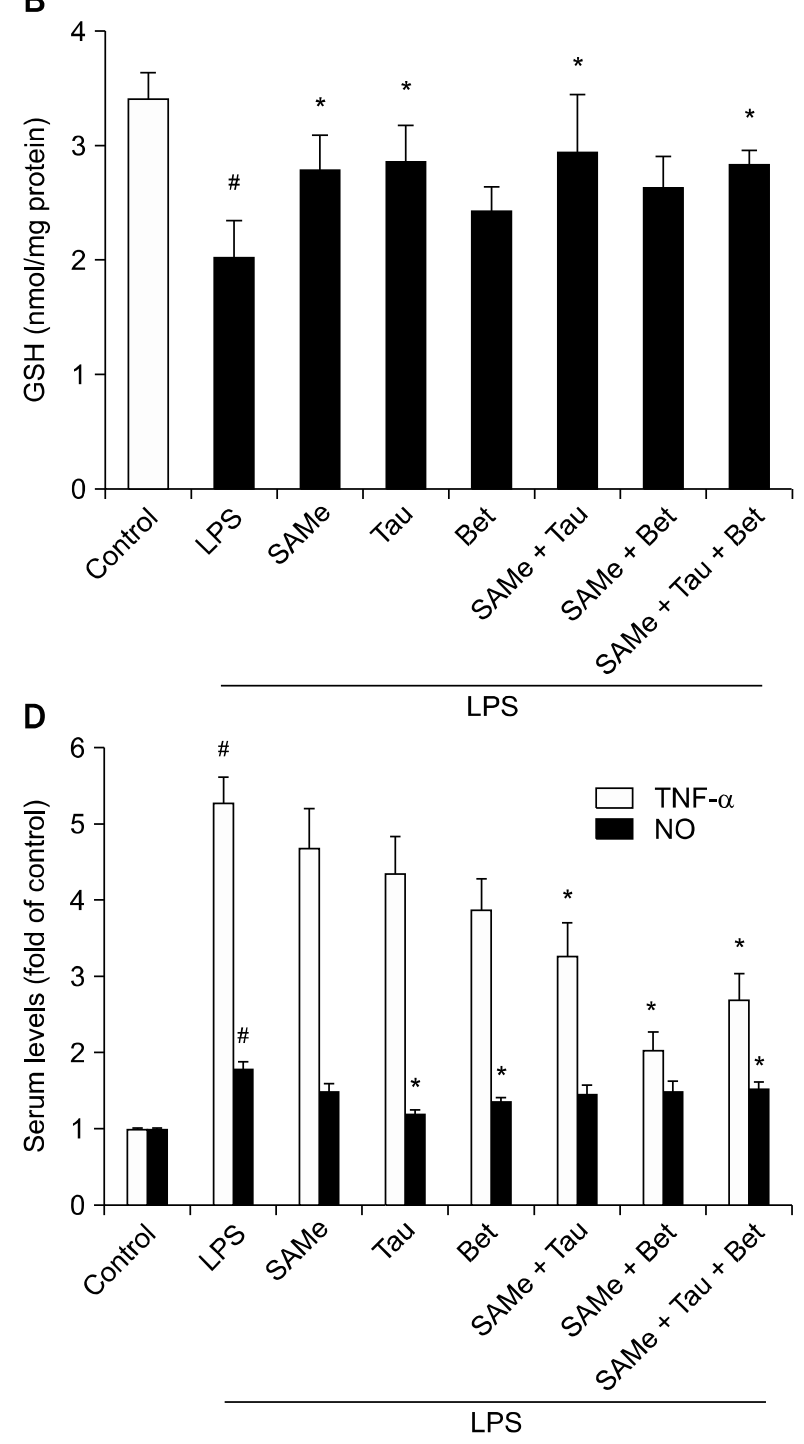

Figure 3. Effects of SAMe, taurine, betaine, and their combinations on serum ALT and AST levels, hepatic GSH levels and mRNA levels of hepatic GSH synthesizing enzymes, and inflammatory mediators in LPS-stimulated mice. (A) Serum ALT and AST levels. (B) Hepatic GSH levels, (C) mRNA levels of hepatic GCLC, GCLM and GS, and (D) NO production was measured as described in Materials and Methods. (D) Serum TNF- $\alpha$ was examined by ELISA. (E) TNF- $\alpha$ and iNOS mRNA levels in LPS-injected mice were measured by RT-qPCR and Hprt1 was used as the housekeeping gene. Each bar represents mean with SEM $(n=5)$. ALT, alanine aminotransferase; AST, aspartate aminotransferase; GSH, glutathione; LPS, lipopolysaccharide; GCLC, glutamate-cysteine ligase catalytic subunit; GCLM, glutamate-cysteine ligase modifier subunit; GS, GSH synthase; NO, nitric oxide; iNOS, inducible nitric oxide synthase; Tau, taurine; Bet, betaine. ${ }^{\#} P<0.01$ vs. control group, ${ }^{*} P<0.01$ vs. LPS group. 
A

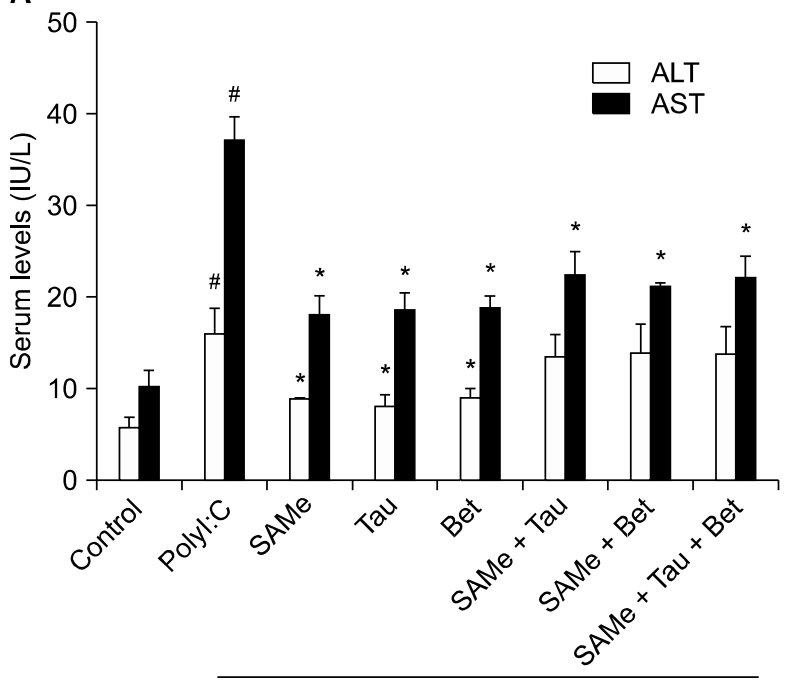

C

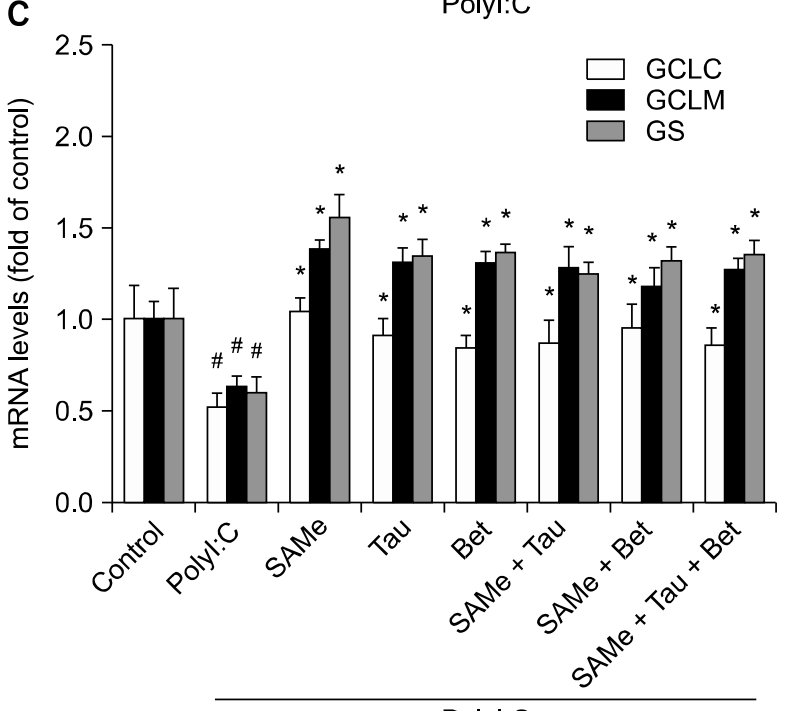

E

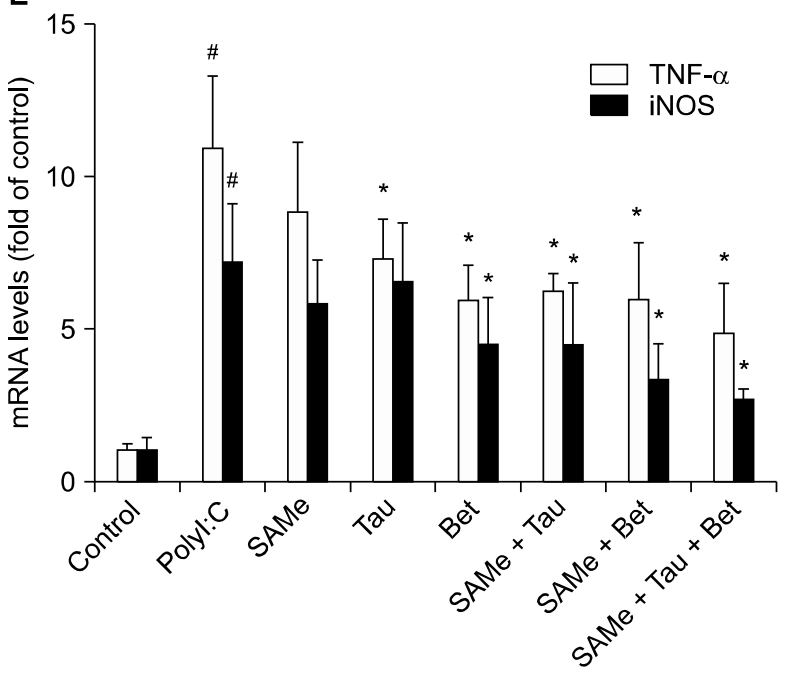

B

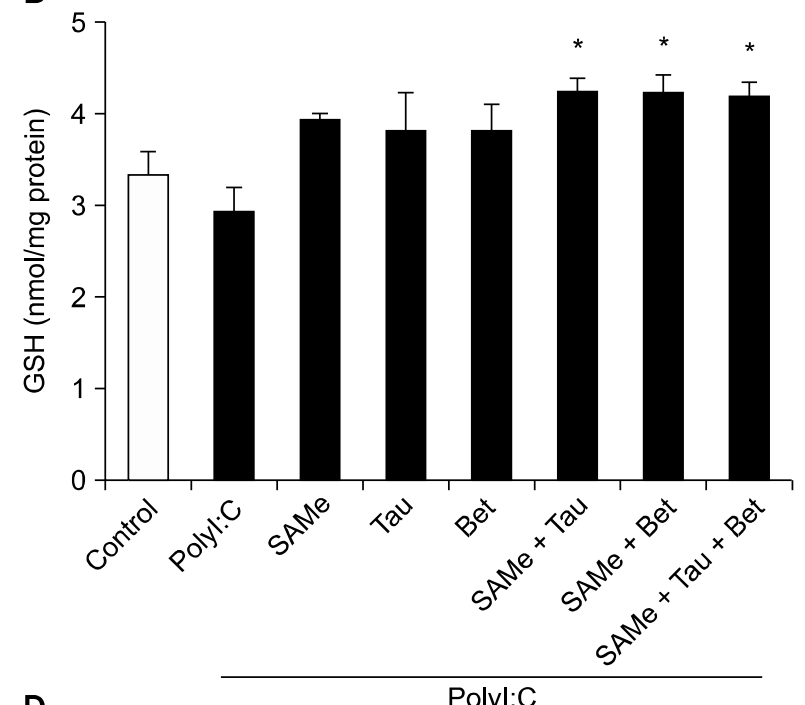

D

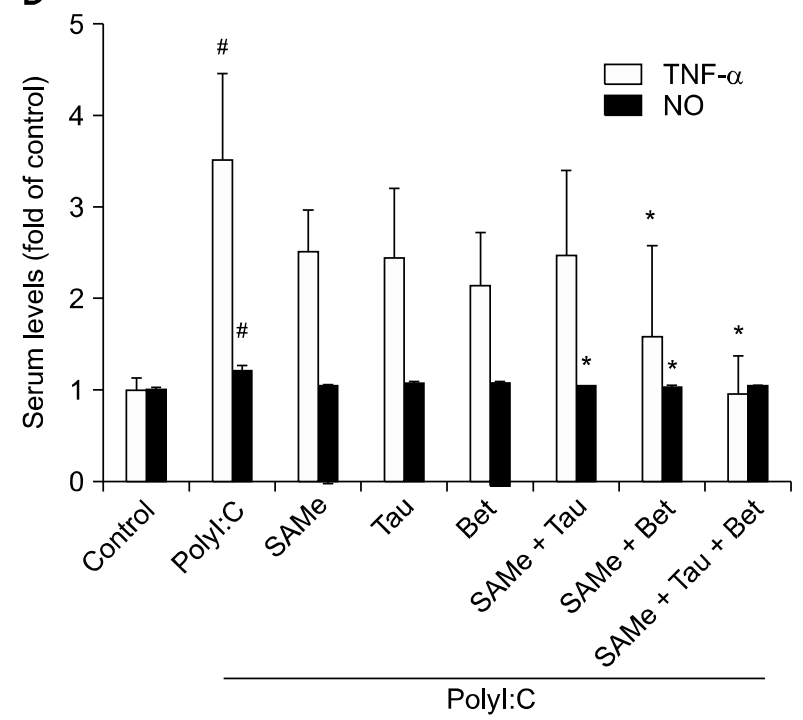

Figure 4. Effects of SAMe, taurine, betaine, and their combinations on serum ALT and AST levels, hepatic GSH levels and mRNA levels of hepatic GSH synthesizing enzymes and inflammatory mediators in polyl:C-stimulated mice. (A) Serum ALT and AST levels, (B) hepatic GSH levels and (C) mRNA levels of hepatic GCLC, GCLM and GS were measured as described in Materials and Methods, (D) NO production and serum TNF- $\alpha$ were examined by Griess reaction assay and ELISA, respectively. (E) TNF- $\alpha$ and iNOS mRNA levels were measured by RT-qPCR and Hprt1 was used as the housekeeping gene. Each bar represents mean with SEM $(n=5)$. ALT, alanine aminotransferase; AST, aspartate aminotransferase; GSH, glutathione; PolyI:C, polyinosinic-polycytidylic acid; GCLC, glutamate- cysteine ligase catalytic subunit; GCLM, glutamate-cysteine ligase modifier subunit; GS, GSH synthase; NO, nitric oxide: iNOS, inducible nitric oxide synthase; Tau, taurine; Bet, betaine. ${ }^{\#} P<0.01$ vs. control group, ${ }^{*} P<0.01$ vs. Polyl:C-treated group. 
pathways, the aim of this study was to evaluate the protective effects of these components and their combinations in LPS- or polyI:C- treated cells and mice.

GSH protects the liver against oxidative stress and influences its susceptibility for damage. ${ }^{28}$ Among the changes in hepatotoxicity, the decrease in GSH level is probably a key protective mechanism. We observed the mRNA expression levels of the GCL and GS. GCL, which is the rate-limiting enzyme, has a catalytic and a modifier subunit, GCLC and GCLM. ${ }^{34}$ The results of our experiments showed that LPS treatment significantly decreased GSH concentration compared to untreated control mice. It has been reported that LPS treatment inactivates hepatic MAT activity. ${ }^{35}$ Because activity of MAT is decreased in patients with liver disease, biosynthesis of hepatic SAMe is reduced and then hepatic GSH level is decreased. ${ }^{36}$ SAMe pretreatment restored hepatic GSH level and mRNA level of GCLC in our in vitro and in vivo experiments. Consistent with this, Ko et al. ${ }^{37}$ has shown the same effects of SAMe pretreatment on GCLC, GCLM and GS expression during endotoxemia. This action of SAMe may be one of the mechanisms for hepatoprotection. Taurine or betaine treatment also tended to prevent the decrease in hepatic GSH levels. Betaine supplementation attenuated the decrease in GSH level in mice and rats. ${ }^{38}$ Therefore, we suggest that the effects of SAMe, taurine and betaine against liver injury might be associated with their regulatory role in the conservation of hepatic GSH level.

Given the importance of GSH in the modulation of liver damage, the effects of SAMe combinations with taurine and/or betaine on cellular GSH level were also evaluated in polyI:Ctreated RAW 264.7 cells and mice. The effects of SAMe and its combinations on virus-infected macrophages are not fully reported. Therefore, we used polyI:C-stimulated macrophages and polyI:C-injected mice to assess the anti-viral capacity of SAMe and its combinations with taurine and/or betaine. To our current knowledge, this is the first report examining whether polyI: $\mathrm{C}$ has influence on GSH levels. PolyI:C treatment downregulated mRNA levels of GCLC, GCLM and GS in mice. We found that SAMe pretreatment and its combinations completely prevented the decrease in GSH level and attenuated the decrease in GCLC, GCLM, and GS mRNA expression in polyI:C-treated RAW 264.7 cells and mice.

When liver function is impaired, endotoxemia can occur and contribute to liver injury. ${ }^{3}$ As in many liver diseases, the inflammation induced by viral infection could accelerate the progression of liver disease. ${ }^{39}$ Pro-inflammatory cytokines such as TNF- $\alpha$ play important roles in liver injury. ${ }^{40}$ TNF- $\alpha$ is involved in immune and inflammatory processes. However, inappropriately overexpressed TNF- $\alpha$ correlates with the development of liver disease during inflammation. ${ }^{41}$ Several studies showed that inflammatory mediators, such as TNF- $\alpha$, stimulate tumor growth and progression in models of cancer and cancer cells. ${ }^{42}$ Another important inflammatory mediator is iNOS. Increased expression of iNOS is followed by excessive NO production. ${ }^{43}$ Since RAW 264.7 cells have been used as an excellent model of inflammatory response in macrophages, LPSor polyI:C-stimulated RAW 264.7 cells were used to evaluate the expression of inflammatory mediators. ${ }^{29}$ In our studies, LPS or polyI:C treatment triggered mRNA expression of iNOS and TNF- $\alpha$ in RAW 264.7 cells. In addition, LPS or polyI:C injection also stimulated an increase in release of NO and TNF- $\alpha$, and mRNA expression of iNOS and TNF- $\alpha$. Other studies have shown that pretreatment with SAMe dramatically inhibited induction of TNF- $\alpha$ and iNOS expression in LPS-stimulated RAW 264.7 cells. $^{44,45}$ However, we and Ara et al. ${ }^{29}$ showed that SAMe treatment did not completely inhibit an LPS-induced increase in TNF- $\alpha$ mRNA expression. Although TNF- $\alpha$ mRNA level was not completely inhibited, SAMe pretreatment significantly lowered TNF- $\alpha$ mRNA expression in LPS- or polyI:C-stimulated RAW 264.7 cells. Our experiments showed that SAMe did not significantly prevent the increase in iNOS mRNA expression both in vitro and in vivo. Likewise, Ko et al. ${ }^{37}$ reported that SAMe treatment did not inhibit the LPS-induced iNOS expression in mice. On the other hand, betaine or taurine treatment dramatically decreased mRNA expressions of iNOS in LPS- or polyI:C-stimulated RAW 264.7 cells and mice. Taurine has been shown as a potent modulator of the pro- inflammatory and immune response. ${ }^{21}$ Other studies reported that taurine had a protective effect against toxins such as arsenic and fluoride in murine hepatocytes. ${ }^{46,47}$ However, the underlying mechanism of its anti-inflammatory activity remains unclear. Betaine was shown to contribute to various diseases caused by inflammation. ${ }^{48}$ Jung et al. ${ }^{38}$ showed that betaine supplementation completely blocked the induction of TNF- $\alpha$ and iNOS mRNA expressions in rats. Betaine has recently been shown to inhibit inflammatory cytokines through inhibition of TLR4 expression or suppression of the activation of NF- $\mathrm{KB}$, which regulates the transcription of pro-inflammatory mediators including TNF- $\alpha$ and iNOS. ${ }^{49.50}$ In this regard, our results suggest that the hepatoprotective mechanism of betaine may be associated with the inhibition of inflammatory factors.

In summary, our study demonstrated that SAMe pretreatment tended to prevent the decrease in GCLC mRNA expression, helping to preserve GSH levels in LPS- or polyI:C-treated RAW 
264.7 cells and mice. This may be a major mechanism of the protective effect of SAMe. Taurine or betaine pretreatment significantly prevented overexpression of pro-inflammatory cytokines and inflammatory mediators in LPS- or polyI:C-treated RAW 264.7 cells and mice. Taken together, SAMe combination with taurine and betaine can protect the liver against LPS- or polyI:C-induced liver injury, most probably via its effects on oxidative stress and inflammatory mediators. Although further work is needed to elucidate the mechanisms of SAMe, taurine and betaine, our study showed the potential effects of SAMe in combination with taurine and betaine on GSH levels and inflammatory mediators. Ultimately, SAMe combinations could have anti-viral, anti-bacterial and hepatoprotective effects, which eventually would contribute to preventing the progression of various liver diseases into liver cancer.

\section{CONFLICTS OF INTEREST}

No potential conflicts of interest were disclosed.

\section{REFERENCES}

1. Gao B, Jeong WI, Tian Z. Liver: an organ with predominant innate immunity. Hepatology 2008;47:729-36.

2. Levrero M. Viral hepatitis and liver cancer: the case of hepatitis C. Oncogene 2006;25:3834-47.

3. Su GL. Lipopolysaccharides in liver injury: molecular mechanisms of Kupffer cell activation. Am J Physiol Gastrointest Liver Physiol 2002;283:G256-65.

4. Oliva J, Bardag-Gorce F, Li J, French BA, French SW. S-adenosylmethionine prevents the up regulation of Toll-like receptor (TLR) signaling caused by chronic ethanol feeding in rats. Exp Mol Pathol 2011;90:239-43.

5. Visintin A, Mazzoni A, Spitzer JH, Wyllie DH, Dower SK, Segal DM. Regulation of Toll-like receptors in human monocytes and dendritic cells. J Immunol 2001;166:249-55.

6. Zhang C, Walker LM, Hinson JA, Mayeux PR. Oxidant stress in rat liver after lipopolysaccharide administration: effect of inducible nitric-oxide synthase inhibition. J Pharmacol Exp Ther 2000;293:968-72.

7. Hansen J, Cherwitz DL, Allen JI. The role of tumor necrosis factor-alpha in acute endotoxin-induced hepatotoxicity in ethanol-fed rats. Hepatology 1994;20:461-74.

8. Alexopoulou L, Holt AC, Medzhitov R, Flavell RA. Recognition of double-stranded RNA and activation of NF-kappaB by Toll-like receptor 3. Nature 2001;413:732-8.

9. Wang N, Liang Y, Devaraj S, Wang J, Lemon SM, Li K. Toll-like receptor 3 mediates establishment of an antiviral state against hepatitis C virus in hepatoma cells. J Virol 2009;83:9824-34.

10. Takii Y, Nakamura M, Ito M, Yokoyama T, Komori A, ShimizuYoshida Y, et al. Enhanced expression of type I interferon and toll-like receptor-3 in primary biliary cirrhosis. Lab Invest 2005; 85:908-20
11. Bigger JE, Thomas CA 3rd, Atherton SS. NK cell modulation of murine cytomegalovirus retinitis. J Immunol 1998;160:5826-31.

12. Ware CF. Network communications: lymphotoxins, LIGHT, and TNF. Annu Rev Immunol 2005;23:787-819.

13. Ulevitch RJ, Tobias PS. Receptor-dependent mechanisms of cell stimulation by bacterial endotoxin. Annu Rev Immunol 1995; 13:437-57.

14. Duce AM, Ortíz P, Cabrero C, Mato JM. S-adenosyl-L-methionine synthetase and phospholipid methyltransferase are inhibited in human cirrhosis. Hepatology 1988;8:65-8.

15. Li K, Chen Z, Kato N, Gale M Jr, Lemon SM. Distinct poly(I-C) and virus-activated signaling pathways leading to interferon-beta production in hepatocytes. J Biol Chem 2005;280:16739-47.

16. Kim SJ, Lee JW, Jung YS, Kwon DY, Park HK, Ryu CS, et al. Ethanol-induced liver injury and changes in sulfur amino acid metabolomics in glutathione peroxidase and catalase double knockout mice. J Hepatol 2009;50:1184-91.

17. Lu SC. Glutathione synthesis. Biochim Biophys Acta 2013;1830: 3143-53.

18. Purohit V, Abdelmalek MF, Barve S, Benevenga NJ, Halsted CH, Kaplowitz N, et al. Role of S-adenosylmethionine, folate, and betaine in the treatment of alcoholic liver disease: summary of a symposium. Am J Clin Nutr 2007;86:14-24.

19. Mato JM, Corrales FJ, Lu SC, Avila MA. S-adenosylmethionine: a control switch that regulates liver function. FASEB J 2002;16: 15-26.

20. Colell A, García-Ruiz C, Miranda M, Ardite E, Marí M, Morales A, et al. Selective glutathione depletion of mitochondria by ethanol sensitizes hepatocytes to tumor necrosis factor. Gastroenterology 1998; 115:1541-51

21. Redmond HP, Stapleton PP, Neary P, Bouchier-Hayes D. Immunonutrition: the role of taurine. Nutrition 1998;14:599-604.

22. Cooper A, Betts JM, Pereira GR, Ziegler MM. Taurine deficiency in the severe hepatic dysfunction complicating total parenteral nutrition. J Pediatr Surg 1984;19:462-6.

23. Mårtensson J, Foberg U, Fryden A, Schwartz MK, Sörbo B, Weiland O. Sulfur amino acid metabolism in hepatobiliary disorders. Scand J Gastroenterol 1992;27:405-11.

24. Lu SC. Regulation of glutathione synthesis. Mol Aspects Med 2009;30:42-59.

25. Barak AJ, Beckenhauer HC, Kharbanda KK, Tuma DJ. Chronic ethanol consumption increases homocysteine accumulation in hepatocytes. Alcohol 2001;25:77-81.

26. Barak AJ, Beckenhauer HC, Mailliard ME, Kharbanda KK, Tuma DJ. Betaine lowers elevated s-adenosylhomocysteine levels in hepatocytes from ethanol-fed rats. J Nutr 2003;133:2845-8.

27. Kim SK, Kim YC. Effects of betaine supplementation on hepatic metabolism of sulfur-containing amino acids in mice. J Hepatol 2005:42:907-13.

28. Payabvash S, Ghahremani MH, Goliaei A, Mandegary A, Shafaroodi H, Amanlou M, et al. Nitric oxide modulates glutathione synthesis during endotoxemia. Free Radic Biol Med 2006; 41:1817-28.

29. Ara AI, Xia M, Ramani K, Mato JM, Lu SC. S-adenosylmethionine inhibits lipopolysaccharide-induced gene expression via modulation of histone methylation. Hepatology 2008;47:1655-66.

30. Wang Y, Xie J, Wang N, Li Y, Sun X, Zhang Y, et al. Lactobacillus casei Zhang modulate cytokine and toll-like receptor expression and beneficially regulate poly I:C-induced immune responses in 
RAW264.7 macrophages. Microbiol Immunol 2013:57:54-62.

31. Reitman S, Frankel S. A colorimetric method for the determination of serum glutamic oxalacetic and glutamic pyruvic transaminases. Am J Clin Pathol 1957;28:56-63.

32. Kwon DY, Jung YS, Kim SJ, Park HK, Park JH, Kim YC. Impaired sulfur-amino acid metabolism and oxidative stress in nonalcoholic fatty liver are alleviated by betaine supplementation in rats. J Nutr 2009;139:63-8.

33. Balkan J, Doğru-Abbasoğlu S, Kanbağli O, Cevikbaş U, Aykaç-Toker $\mathrm{G}$, Uysal M. Taurine has a protective effect against thioacetamideinduced liver cirrhosis by decreasing oxidative stress. Hum Exp Toxicol 2001;20:251-4.

34. Huang CS, Chang LS, Anderson ME, Meister A. Catalytic and regulatory properties of the heavy subunit of rat kidney gamma-glutamylcysteine synthetase. J Biol Chem 1993;268:19675-80.

35. Avila MA, Mingorance J, Martínez-Chantar ML, Casado M, Martin-Sanz P, Boscá L, et al. Regulation of rat liver S-adenosylmethionine synthetase during septic shock: role of nitric oxide. Hepatology 1997;25:391-6.

36. Mato JM, Lu SC. Role of S-adenosyl-L-methionine in liver health and injury. Hepatology 2007;45:1306-12.

37. Ko K, Yang H, Noureddin M, Iglesia-Ara A, Xia M, Wagner C, et al. Changes in S-adenosylmethionine and GSH homeostasis during endotoxemia in mice. Lab Invest 2008:88:1121-9.

38. Jung YS, Kim SJ, Kwon do Y, Ahn CW, Kim YS, Choi DW, et al. Alleviation of alcoholic liver injury by betaine involves an enhancement of antioxidant defense via regulation of sulfur amino acid metabolism. Food Chem Toxicol 2013;62:292-8.

39. Yuan L, Kaplowitz N. Glutathione in liver diseases and hepatotoxicity. Mol Aspects Med 2009;30:29-41.

40. Tracey KJ, Cerami A. Tumor necrosis factor: a pleiotropic cytokine and therapeutic target. Annu Rev Med 1994:45:491-503.
41. Moro C, Palacios I, Lozano M, D’Arrigo M, Guillamón E, Villares A, et al. Anti-inflammatory activity of methanolic extracts from edible mushrooms in LPS activated RAW 264.7 macrophages. Food Chem 2012;130:350-5.

42. Lin WW, Karin M. A cytokine-mediated link between innate immunity, inflammation, and cancer. J Clin Invest 2007;117:117583.

43. MacMicking JD, Nathan C, Hom G, Chartrain N, Fletcher DS, Trumbauer M, et al. Altered responses to bacterial infection and endotoxic shock in mice lacking inducible nitric oxide synthase. Cell 1995;81:641-50.

44. Majano PL, García-Monzón C, García-Trevijano ER, Corrales FJ, Cámara J, Ortiz P, et al. S-Adenosylmethionine modulates inducible nitric oxide synthase gene expression in rat liver and isolated hepatocytes. J Hepatol 2001;35:692-9.

45. Watson WH, Zhao Y, Chawla RK. S-adenosylmethionine attenuates the lipopolysaccharide-induced expression of the gene for tumour necrosis factor alpha. Biochem J 1999;342:21-5.

46. Sinha M, Manna P, Sil PC. Taurine, a conditionally essential amino acid, ameliorates arsenic-induced cytotoxicity in murine hepatocytes. Toxicol In Vitro 2007;21:1419-28.

47. Das J, Ghosh J, Manna P, Sil PC. Taurine provides antioxidant defense against NaF-induced cytotoxicity in murine hepatocytes. Pathophysiology 2008;15:181-90.

48. Craig SA. Betaine in human nutrition. Am J Clin Nutr 2004:80: 539-49.

49. Yi EY, Kim YJ. Betaine inhibits in vitro and in vivo angiogenesis through suppression of the NF- $\kappa \mathrm{B}$ and Akt signaling pathways. Int J Oncol 2012;41:1879-85.

50. Shi QZ, Wang LW, Zhang W, Gong ZJ. Betaine inhibits toll-like receptor 4 expression in rats with ethanol-induced liver injury. World J Gastroenterol 2010;16:897-903. 